\title{
Transposition
}

Musique et Sciences Sociales

9| 2021

Musique et sexualité

\section{Barbara L. Kelly, Christopher Moore (eds.), Music Criticism in France, 1918-1939. Authority, Advocacy, Legacy}

Woodbridge, The Boydell Press, 2018

Florence Huybrechts

\section{CpenEdition}

Journals

Édition électronique

URL : http://journals.openedition.org/transposition/5517

DOI : 10.4000/transposition.5517

ISSN : $2110-6134$

Éditeur

CRAL - Centre de recherche sur les arts et le langage

Référence électronique

Florence Huybrechts, «Barbara L. Kelly, Christopher Moore (eds.), Music Criticism in France, 1918-1939.

Authority, Advocacy, Legacy», Transposition [En ligne], 9 | 2021, mis en ligne le 15 décembre 2020, consulté le 23 avril 2021. URL : http://journals.openedition.org/transposition/5517 ; DOI : https:// doi.org/10.4000/transposition.5517

Ce document a été généré automatiquement le 23 avril 2021

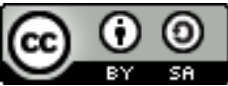

La revue Transposition est mise à disposition selon les termes de la Licence Creative Commons Attribution - Partage dans les Mêmes Conditions 4.0 International. 


\section{Barbara L. Kelly, Christopher Moore (eds.), Music Criticism in France, 1918-1939. Authority, Advocacy, Legacy}

Woodbridge, The Boydell Press, 2018

Florence Huybrechts

\section{RÉFÉRENCE}

Barbara L. Kelly, Christopher Moore (eds.), Music Criticism in France, 1918-1939. Authority, Advocacy, Legacy, Woodbridge, Boydell Press, 2018, 358 p.

1 Résultat d'un workshop international (Ottawa, 2011) unissant les efforts de chercheurs de pays et d'horizons disciplinaires variés, ce recueil de 12 études se veut une première enquête approfondie sur la critique et les critiques musicaux de l'entre-deux-guerres français. Il témoigne de l'engouement récent ayant présidé, de part et d'autre de l'Atlantique, à la formation de collectifs de recherche, lesquels ont contribué à faire passer la presse et la critique musicales françaises d'un réservoir de connaissances empiriques à un objet d'étude à part entière - citons le réseau «Francophone Music Criticism Network 1789-1914» (soutenu par l'Arts and Humanities Research Council), l'équipe de recherche «Musique en France au $\mathrm{XIX}^{\mathrm{e}}$ et $\mathrm{xx}^{\mathrm{e}}$ siècles: discours et idéologies » (à l'observatoire interdisciplinaire de création et de recherche en musique de l'Université de Montréal), et le programme «La critique musicale au $\mathrm{xx}^{\mathrm{e}}$ siècle » (Institut universitaire de France). Dans sa globalité, l'ouvrage exploite un riche corpus d'écrits critiques et de documents d'archives, à travers lesquels il entend interroger à la fois l'histoire des idées et la culture musicale des années 1918 à 1939. Les éditeurs ont fait le choix d'une organisation implicite selon trois thématiques interconnectées - authority, advocacy, legacy -, que nous ne suivrons que partiellement pour épingler les principaux apports du volume. 
2 Une question centrale que pose l'ensemble est celle de la légitimité des différentes pratiques de la critique musicale. C'est, en premier lieu, celle que s'arrogent de nouveaux standards d'écriture, favorisés par le développement de la musicologie et la montée en puissance de la voix des compositeurs; les attitudes contrastées que les "spécialistes » adoptent face à la question de l'expertise technique, et la présence toujours sensible des écrivains et autres "musicographes" au sein de la presse musicale, aiguisent le clivage entre une critique-œuvre littéraire (ou subjective) et une critique-œuvre scientifique (objective), comme le rappelle utilement la contribution inaugurale de Michel Duchesneau (p. 17-42). La légitimité, ce peut être aussi le capital symbolique et le degré de reconnaissance acquis ou convoités par le critique dans l'exercice de son métier. Une série d'articles se concentrent ainsi sur les trajectoires professionnelles de critiques musicaux, et sur les stratégies qu'ils déploient à la conquête d'une position médiatique enviable. Philippe Cathé (p.63-90) analyse les motivations de l'activité exégétique de Koechlin, et examine les étapes de la construction d'une autorité reposant sur les ethè de compositeur, de pédagogue et d'intellectuel engagé. Kimberly Francis (p. 169-191) envisage les colonnes signées par Nadia Boulanger dans Le Monde Musical en ce qu'elles illustrent l'expérience d'une femme dans le monde musical de l'immédiat après-guerre; elle décèle un mode singulier, chez la musicienne, de se positionner dans la communauté critique : ainsi une insertion habile dans les débats du moment (Vincent d'Indy vs Claude Debussy, Maurice Ravel vs Erik Satie) et une forte pondération dans le jugement, qui trahit l'urgence de s'assurer une stabilité professionnelle.

3 L'ouvrage interroge aussi très largement l'intervention de la critique dans les processus d'attribution de la valeur musicale, et la reconfiguration, face à de nouveaux enjeux esthétiques, du conflit entre "modernistes » et «traditionalistes » qui avait secoué les premières années du siècle. Dans une part non négligeable des corpus critiques soumis à l'analyse, la défense active d'une génération musicale (celle qui est établie, celle qui demande à l'être) peut révéler des ambitions manifestaires ou des logiques d'autopromotion sous-jacentes. Pascal Lécroart (p.151-167) étudie quelques articles signés par Arthur Honegger dans Musique et Théâtre (1925-1926), au travers desquels le compositeur élabore une poétique du drame lyrique. En sondant les chroniques musicales qu'Alejo Carpentier a livrées à des journaux cubains et parisiens des années 1922 à 1939, Caroline Rae (p. 193-217) démontre que la peinture et la promotion de l'avant-garde musicale parisienne peuvent être un moyen d'asseoir la construction d'une identité musicale latino-américaine. Jacinthe Harbec (p. 267-292) examine les raisons de la réception tantôt mitigée, tantôt franchement critique, des musiques de Relâche (ballet signé Francis Picabia, Erik Satie et René Clair). Aux côtés des critiques qui saluent ou égratignent l'avant-garde, de nombreux exégètes s'attachent à consolider une attitude critique héritée d'avant-guerre, en portant aux nues la «trinité » musicale que forment Gabriel Fauré, Claude Debussy et Maurice Ravel. Les contributeurs de l'ouvrage sont plusieurs à explorer le rôle de ces «agents de canonisation » dans la polarisation du champ critique français des années 1918-1939. Plus particulièrement, Marianne Wheeldon (p. 219-243) emprunte à Gary Fine la notion de reputational entrepreneur pour caractériser l'action de quatre critiques (Émile Vuillermoz, Charles Koechlin, Louis Laloy et Léon Vallas) qui ont participé à minimiser le revers de fortune de Claude Debussy après 1918; elle montre que cette campagne doit beaucoup à la volonté de ces hommes de (re)négocier leur propre position professionnelle dans l'environnement musical parisien d'après-guerre. Avec un angle 
plus étroit, Danick Trottier (p. 293-315) interroge le système de valeurs construit par Musiques d'aujourd'hui (1923), une compilation d'articles de presse où Émile Vuillermoz, rétif aux hardiesses du Groupe des Six, réaffirme les compositeurs déjà cités au rang de piliers de la modernité musicale.

De nombreuses contributions du volume rendent assez fidèlement compte de ce que la construction de ces canons musicaux reflète de fortes préoccupations idéologiques. Les chercheurs identifient dans la production critique un nuancier de positions, des plus internationalistes (Henry Prunières, Charles Koechlin, Nadia Boulanger, Alejo Carpentier) aux plus étroitement chauvines. Se penchant sur ces dernières, Christopher Moore (p. 43-61) explore la contribution de Dominique Sordet et de Lucien Rebatet au quotidien L'Action française: une rubrique musicale à deux voix, fidèle aux valeurs antidémocratiques et au nationalisme intégral de Charles Maurras, étayant l'ethos nostalgique de l'un, une esthétique de la violence (et un féroce antisémitisme) chez l'autre. Jann Pasler (p. 91-119) perçoit à travers l'entreprise collective de L'initiation à la musique à l'usage des amateurs de musique et de radio (1935), l'héritage d'une idéologie «bleu horizon» (remontant aux idéaux unionistes de la jeune III République), qui traduirait l'« alliance " sacrée de la critique des années 1930 : manière de concevoir ce qui peut rassembler des contributeurs d'orientations politiques variées (Maurice Emmanuel, Reynaldo Hahn, Paul Landormy, Émile Vuillermoz, Dominique Sordet, etc.) dans un engagement unanime pour la musique contemporaine française et une même ouverture aux nouvelles technologies. Dans l'une des contributions les plus originales de l'ouvrage (p. 121-149), Barbara L. Kelly envisage elle aussi la rencontre d'opinions divergentes - le nationalisme fervent de Léon Vallas et les idéaux internationalistes d'Henry Prunières - dans la promotion du canon musical français; elle s'interroge surtout sur les relais institutionnels de la presse musicale, et définit les cycles de concerts organisés par les deux hommes (les Concerts de la Revue musicale et Les Petits Concerts) comme un canal alternatif de leur entreprise critique, et l'extension performative de leur production écrite. Rachel Moore (p. 245-265) analyse quant à elle une série de rétrospectives consacrées à la vie musicale française durant la Grande Guerre, et plus spécifiquement deux séries signées par Julien Tiersot et Arthur Pougin dans Le Ménestrel (1919 et 1920-21) ; contre l'idée d'un " hiatus musical », ces ensembles documentaires façonnent la vision idéale d'un Paris musical revenu à la «vie normale ", et dessinent une efficace propagande d'après-guerre, qui participe à la fois d'un effort de reconstruction morale de la patrie, et d'un agenda nationaliste destiné à endiguer la progression des influences musicales étrangères.

Les prises de position individuelles et collectives, les parcours et trajectoires abordés dans ce volume laissent entrevoir les fonctions plus ou moins assumées de l'activité critique (nécessités financières, autopromotion, didactique compositionnelle, plaidoyer esthétique ou politique) et, d'une manière générale, invitent à concevoir la pratique du commentaire et l'esthétique musicale de l'entre-deux-guerres en termes d'héritage et de dépassement, de continuités et de ruptures. Arrivée à maturité après 1918, dépassant pour partie des jeux de chapelles qu'une complexification des langages esthétiques tend à estomper, la critique musicale française subit dans le même temps une série de bouleversements dont Michel Duchesneau offre la synthèse - il y a, outre l'opposition entre ses différents corps de métier, une politisation croissante du discours, et un déplacement des principales fonctions culturelles qui lui sont attachées :

[...] music criticism appeared to withdraw progressively from daily musical life; it

became more specialised and abandoned the evaluation of concert life in favour of 
discussions of works rendered more 'permanent' via the technologies of the disc and radio. It thus no longer focused on what the work IS, but on its REPRODUCTION and, consequently, on those who reproduced it - the performers. (p. 20) critique singulier qu'ils appellent (effleurée chez Philippe Cathé et Jann Pasler) sont précisément l'une des problématiques dont on peut regretter qu'elles ne soient davantage présentes dans l'ouvrage, quand l'introduction laissait présager un traitement à part entière. De la même façon, si le décryptage des enjeux politiques inhérents à la formation des canons musicaux constitue l'une des lignes directrices du volume, on peut s'étonner de l'absence radicale des musiques non canoniques (jazz, music-hall, folklore), dont la critique (éminemment politique elle aussi) émerge et se structure à l'époque définie. À l'une ou l'autre exception près, les corpus sont abordés sous l'angle monographique ; l'échantillon favorise du reste les commentateurs fameux dont la production a souvent déjà fait l'objet d'analyses et d'éditions critiques (Charles Koechlin, Émile Vuillermoz, Boris de Schlœzer). Mais sans doute est-ce le propre d'un travail collectif de composer un savoir fragmentaire; le lot, aussi, d'un domaine de recherche en pleine construction, qui fournira tôt ou tard les données historicostatistiques nécessaires à l'établissement d'une vision globale de champs critiques tels qu'en contexte.

7 La démarche sera rendue possible à condition qu'un réel dialogue se noue entre les traditions musicologiques française et anglo-saxonne. Si l'impression nous reste que la sauce peine à prendre (à la lecture d'un préambule qui consacre l'heureuse union autant qu'il assoit le clivage), on ne peut que saluer la qualité générale, l'apport empirique et la diversité théorique des contributions rassemblées dans cet ouvrage.

\section{AUTEURS}

\section{FLORENCE HUYBRECHTS}

Docteure en Langues et lettres de l'Université libre de Bruxelles, Florence Huybrechts a soutenu une thèse intitulée L'écrivain mélomane, entre presse et livre (1919-1939). Pratiques, éthique et genres d'un discours sur la musique (2017, à paraître en 2021). Après des séjours postdoctoraux à Columbia University (New York) et à La Sapienza (Rome), elle entame à l'Université de Lausanne un projet de recherche portant sur l'histoire et l'esthétique du radio-théâtre en Suisse et en Belgique francophones. 